\title{
संगीत एवं योग में अन्तर्सम्बन्ध
}

\section{सार संक्षेपिका}

मन की चंचलता दूर कर उसे स्थिर बनाने वाली श्रेष्ठतम विद्या योग है। तो श्रेष्ठतम कला संगीत। इस प्रकार संगीत और योग का आपस में गहरा सम्बन्ध है। योग के समान संगीत का प्राथमिक विकास भी, आध्यात्मिक विकास के लिए हुआ है। संगीत को ईश्वर का दर्जा प्राप्त है इसलिए इस विधा में शुद्धता और शास्त्रीयता का विशेष महत्व है। सात शुद्ध और पांच कोमल स्वरों के माध्यम से मन को साध लेना ही संगीत है। एक तरफ जहां 'योग' से शरीर, मन, मस्तिष्क को साधा जाता है वहीं संगीत हमारी आत्मा को शुद्ध करता है। संगीत में स्वरों की शुद्धता पर और उसकी पवित्रता पर जोर दिया जाता है, परन्तु योगशास्त्र में आसन व मुद्राओं पर जोर दिया है। दोनों में ही स्वर व मुद्रा की श्रेष्ठता से आनन्द तथा स्वास्थ्य पाया जा सकता है। संगीत और योग दोनों विधाएं अलग होने के बावजूद भी ये एक-दूसरे के पूरक हैं। दोनों के मूल तत्त्व और परिणाम सार्वकालिक है। दोनों का उद्देश्य आत्मा का परमात्मा से मिलन है।

बीज शब्द

लोक कला, संगीत, योग

भूमिका

संगीत सृष्टि के कण-कण में व्याप्त है। संगीत मनुष्य से लेकर जीव-जन्तु पेड़-पौधो, नदियों, झरनों इत्यादि सभी में व्याप्त है। संगीत आत्मा रूपी शरीर को पूर्ण रूप से धारण कर चुका है। जीवन के लिए संगीत उतना ही आवश्यक है, जितना श्वास। संगीत की अपनी ही अनूठी भाषा है, अभिव्यक्ति है, जो बिना शब्दों के एक-दूसरे तक आसानी से पहुँच जाती है। जितना योग करना महत्वपूर्ण है, संगीत का साथ भी उतना ही महत्वपूर्ण है। क्योंकि योग शरीर को स्वस्थ रखता है तो संगीत हृदय और आत्मा को उतना ही संतृप्त करता है। संगीत और योग से जीवन में शक्ति का विकास होता है। "संगीत एक ऐसी विधा है जो प्रत्येक मानव के साथ प्रत्यक्ष रूप से जुड़ी है। संगीत की उत्त्पति का कारण ध्वनि अथवा नाद है।"

\section{गीतं नादात्मकं वाद्यं नाद व्यक्तया प्रशस्यते।}

तद्द्वयानुगतं नृतं नादाधीनमतस्त्रयम।।

गीत नादात्मक है। वाद्य नाद की व्यक्ति के कारण प्रंशसित होता है। नृत्य उन दोनों का अनुगत है। इसलिए तीनों नाद के अधीन है।

नादेन व्यंज्यते वर्ण: पदं वर्णात्पदाद्धचः।

वचसो व्यवहारोयं नादधीनमतो जगत्।।

नाद से वर्ण, वर्ण से पद, पद से वाक्य व्यक्त होता है। वाक्य से यह व्यवहार भाषा बनती है। इसलिए सम्पूर्ण जगत नाद के अधीन है। योग में हम कह सकते हैं "नादबह्म", जिसका अर्थ है "ध्वनि ही ईश्वर है, ऐसा इसलिए क्योंकि जीवन का आधार कम्पन में है यह कम्पन्न ही ध्वनि है। 
नाद बह्म संगीत का उद्गम है। मनुष्य की वाणी नाद का ही स्वरूप है। प्रत्येक श्वास-प्रश्वास में जो गति होती है, वह ताल को अभिव्यक्त करती है। स्वरों की साधना, रियाज, लय, ताल शासत्र की शुद्ध पद्धति के द्वारा नाद बह्म की अराधना कर अंतर्मन में गहराई तक उतरना ही संगीत का मुख्य लक्ष्य है। इसलिए संगीत एंव योग एक-दूसरे से जुड़े हुए हैं। दोनों का उद्देश्य (एकाकार) या आत्मसाक्षात्कार है। योगियों के मत में संगीतमय नाद एक विशिष्ट प्रक्रिया है। जैसे प्राणायाम से चित-वृत्तियों का निरोध होता है, वैसे संगीत से भी होता है। प्राण का सम्बन्ध आकाश एवं वायु तत्वों के साथ है, इसी प्रकार संगीत का सम्बन्ध भी इन तत्वों के साथ है। संगीत का सम्बन्ध नाद से है और नाद का प्राणवहन से। प्राणायाम से तात्पर्य प्राण के आयाम से जिस प्रकार चित्त शांत होता है अर्थात योग में श्वास-प्रश्वास से सुरों की साधना होती है। उसी प्रकार संगीत में सांगीतिक नाद से चित्त शांत होता है अर्थात श्वास पर नियन्त्रण कर सुरों की साधना होती है। इस क्रिया से फेफड़े स्वस्थ होते हैं। साधना करने से गले में होने वाले संक्रमण से बचाव होता है, योग में भी शारीरिक क्रियाओं के माध्यम से तन-मन को स्वस्थ बनाने की कोशिश की जाती है। विभिन्न राग, मन को शन्ति प्रदान करते हैं, वही ब्लड़ प्रेशर कंट्रोल करने में अपनी महत्वपूर्ण भूमिका निभाते है। योग भी तो ऐसी बीमारियों को ठीक करने में अपनी भूमिका निभाता है। आज म्यूजिक थैरेपी को सारी दुनिया मान चुकी है। उदाहरणस्वरूप तबले पर जब वादन किया जाता है तो उसका माध्यम अंगुलियां होती है जिसके वादन से अंगुलियों के जोड़, कोहनी और हाथों का व्यायाम भी हो जाता है। बांसुरी के द्वारा भी जब वादन किया जाता है तो उसे भी योग का हिस्सा माना जाता है क्योंकि बांसुरी वादन में हवा पर नियन्त्रण किया जाता है और श्वास (अन्दर लेना) प्रश्वास जैसी क्रियाएं होती है, जिसे योग की भाषा में प्राणायाम कहते है। और जब हम बांसुरी बजाते हैं तो श्वास पर नियन्त्रण करने के लिए ध्यान लगाना पड़ता है। ध्यान को योग की ही क्रिया माना जाता है।

नादयोग को शास्त्रीय संगीत की दुनिया का योग भी माना जाता है। शास्त्रीय गायन में ब्रह्मनाद को प्राप्त करना है तो इसे नाद योग से प्राप्त किया जाता है। नादयोग का सम्बन्ध भी श्वास की निरन्तरता से होता है नादयोग संगीत की योग क्रिया है, इसके अलावा भी कई स्वर ऐसे है जो नाभि द्वारा लगाए जाते हैं। इन सुरों को लगाने में नाभि पर जो दबाब पड़ता है, उससे जो सधा स्वर निकलता है उसके साथ-साथ कपालभाति के समान ही नाभि का भी व्यायाम हो जाता है। ओंकार साधना के लिए नाभि से स्वर निकालना कपाल भाति योग के समान ही है, संगीत और योग दोनों से एकाग्रता सिद्ध की जाती है

संगीत आत्मा की सात्विकता को लिए हुआ है। बह्म के परम पवित्र प्रणव नाद ओंकार की उत्पत्ति सृष्टि के साथ मानी गई है। भारतीय संगीत के सात स्वर न केवल हमारी शारीरिक तंत्रिकाओं को प्रभावित करते हैं, बल्कि पाशविक वृत्तियों का दमन भी करते हैं। और हमारे अन्दर आध्यात्मिक एवं 
सात्तिक विचारों का संचार भी करते हैं। योग अगर संगीत के साथ किया जाए तो शरीर और आत्मा का मिलन बहुत तीव्र गति से होता है।

संगीतकार की कुंडलिनी शक्ति भी इसी प्रकार जागृत होती है, जिस प्रकार एक योगाभ्यासी की, क्योंकि साधना दोनों में है। योग में आसन, प्राणायाम, मुद्रा ध्यान, धारणा और समाधि सर्वोपरि है। संगीत में स्वर, लय, ताल, साहित्य, भाव और रस प्रमुख है। इन दोनों में ध्यान का स्थान महत्वपूर्ण है। ध्यान द्वारा ही चक्रों और कुंडलिनी शक्ति का जागरण होता है। अगर सांगीतिक भाषा में कहना हो तो चक्रों में होने वाली असांगीतिक लहरें सांगीतिक होने लगती है। भारत के योगियों ने सात स्वरों की उत्पत्ति मानव शरीर के सात यौगिक चक्रों से मानी है। जब स्वरों को गाया-बजाया जाता है, तो स्वरों में जो आन्दोलन अलग-अलग निश्चित संख्या में होता है, उनके कम्पन का प्रभाव शरीर के यौगिक चक्रों पर पड़ता है, इसी स्वर उपासना से मनुष्य आहत नाद से अनाहत नाद की ओर अग्रसर होता है। योगियों की भाषा में संगीत 'नादयोग' है। सात यौगिक चक्र मानव शरीर के सात स्थानों में सर्प की भांति कुंडली मारे हुए है। सात योगिक स्थानों से विकसित शक्तियों को योगी एकत्र करके जैसे-जैसे उर्ध्वगामी करते हैं। वैसे-वैसे शक्ति मनुष्य के नीचे के केन्द्रों से उपर के केन्द्र तक जाती है। अंततः सप्तचक्र भेदन होने पर शिव-शक्ति अर्थात कुण्डलिनी शक्ति जागृत होती है, वही मोक्ष प्राप्ति का साधन है।

स्वर साधना के बल पर प्राचीन काल में तानसेन, बैजू जैसे संगीतकारों ने कुण्डलिनी शक्ति को इतना अधिक जागृत किया कि वह अपने स्वरों के प्रभाव से दीपक जलाने, वर्षा करने तथा पत्थर पिघलाने जैसे चमत्कार किए। यह सब ध्यान, एकाग्रता और समाधि का ही परिणाम है। एकाग्रता इतनी प्रबल होनी चाहिए जितनी अर्जुन द्वारा पानी में देखकर चलती मछली की परछाई द्वारा मछली की आँख बीधना। तभी कुण्डली शक्ति जागृत हो सकती है।

"सशैल वन धात्रीणां यथाधारोहिनायकः।

सर्वेषां योगतन्त्राणां तथाधारो हि कुण्डली"।

अर्थात श्लोक में बताया गया है कि कुण्डलिनी शक्ति सब प्रकार के योगों की आश्रय स्वरूपा है। योगों में संगीत को बह्म योग कहा गया है। जिस प्रकार सर्पों के अधिनायक 'शेष' इस सम्पूर्ण पृथ्वी को अपने फन पर धारण किए हुए है, उसी प्रकार सब प्रकार के योगों को कुण्डलिनी धारण करती है। क्योंकि कुण्डलिनी के जागरण के बिना योग के सभी उपाय निष्फल रहते हैं। हमारे शरीर में योगाचार्यों द्वारा जिन चक्रों तथा नाड़ियों का वर्णन किया गया है। उनसे ही स्वर, व्यंजन तथा वर्ण की उत्पत्ति मानी जाती है। हर चक्र में आरम्भ से लेकर सभी स्वर, व्यजनों का सथान माना गया है। 
संगीत और योग दोनों साधनाओं में अनाहत चक्र के नीचे मणिपुर स्वाधिष्ठान और मूलाधार चक्रों की साधना के महत्व को समान रूप से स्वीकार किया गया है। मूलाधार चक्र के निकटतम निम्न भाग में मूलकंद है और इन्हीं दोनों स्थानों का आश्रय लेकर कुंडलिनी निवास करती है। जब यह कुण्डलिनी जागृत होती है तो यह यह षटचक्रों का भेदन करके साधक को ब्रह्म में विलीन कर देती है। योग के समान संगीत साधना में भी इन चक्रों पर अधिकार करना अपेक्षित माना गया है। जिन सप्त चक्रों से सात स्वरों की उत्पत्ति मानी है वह मूलाधार चक्र है अर्थात सा स्वर की उत्पत्ति मूलाधार चक्र से मानी गई है। मूलाधार चक्र से मानी गई है। मूलाधार चक्र का हमारे शरीर में गुदा स्थान है। जब 'सा' की आन्दोलन संख्या या कम्पन बढ़ती है तो 'रे' स्वर की उत्पत्ति होती है। जिसका केन्द्र स्वाधिष्ठान चक्र माना गया है। 'रे' से रस की उत्पत्ति होने लगती है। स्वाधिष्ठान का स्थान शरीर में जननेन्द्रिय के ठीक उपर और जघन अस्थि पर स्थिर है। स्वाधिष्ठान में वायु को अवरूद्ध करने वाला संगीत साधक मन्द्र, मध्य एवं तार तीनों सप्तकों में इच्छानुसार विचरण करता हुआ संगीत को त्रिगुणातीत बनाकर अपने लिए नए लोक का निर्माण कर लेता है।

गन्धार स्वर की उत्पत्ति मणिपुर चक्र से होती है। गंधार स्वर से ही अग्नि तत्व की जागृति होती है। मणिपुर चक्र का स्थान नाभि में होता है 'संगीत रत्नाकर' के अनुसार जिस साधक को मणिपुर चक्र में वायु अवरूद्ध करने की शक्ति प्राप्त हो जाती है उसके विकृति भाव नष्ट हो जाते हैं और अंतः करण में आनंद के भाव उदित हो जाते हैं।

मध्यम (म) का उत्पत्ति स्थान अनाहत चक्र है, जिसका स्थान वक्ष स्थल के मध्य रीढ़ की हड्डी के अन्दर है। यह चक्र शरीर को दो भागों में बांटता है। मध्यम स्वर एक लय बनाता है, यह लय मंकार कहलाती है जिसके कारण अनाहत नाद की अनुभूति होने लगती है।

'संगीत रत्नाकर' के अनुसार अनाहत चक्र के उपर विशुद्धि चक्र है, जो पंचम स्वर का उद्गम स्थान है। जिसका स्थान गले में (थाइराइड) के पास होता है। प अर्थात् प्राण, विशुद्ध चक्र में 16 (सोलह) पत्र हैं जिसका नाम 'सोमचक्र' है। यही चक्र सरस्वती को निवास स्थान है। इसमें सोलह पत्र हैं उन पत्रों पर क्रमशः ओंकार, उद्गीथ, हुंकार, वणठ, स्वधा, स्वाहा, ब्रह्मा, आत्मा, षड़ज, ऋषभ, गंधार, मध्यम, पंचम धेवत, निषाद और विष का निवास है। यह सभी भाव अमूर्त है।

प्रथम से सप्तम दल के भाव देव आदि कार्यों से सम्बन्धित हैं। नवम से पन्द्रहवें दल के भाव संसार से। इसमें अष्टम दल पर विराजमान संगीत साधक को आत्मा जब आलौकिक एवं लौकिक, दोनों भावों की ओर समान रूप से देखने लगती है तब उसके लिए विष (संसार) अनायास समाप्त हो जाता है। इस प्रकार यह सोलह पत्र प्राण वायु को नियन्त्रित करते हैं, और यह पत्र साधना से ही संस्कारित होते हैं। वायु नियन्त्रण के कारण ही संगीत के स्वर अपनी श्रुतियों पर स्थिर रह पाते 
है। पंचम स्वर की उत्पत्ति होने के बाद मनुष्य ध्यान की ओर बढ जाता है, उसी ध्यान में जब वह लीन हो जाता है तो आज्ञा चक्र से धैवत (ध) स्वर की उत्पत्ति होती है और व्यक्ति अंतर्मुखी हो जाता है। आज्ञा चक्र का स्थान दोनों भौहों के बीच में है। जहां पर तिलक लगाया जाता है। यहां पर भगवान शिव (रूद्र ग्रन्थि) का निवास स्थान कहा जाता है। इसी धैवत के ध्यान में आज्ञा की जागृति से सूरदास, हरिदास जैसे महान कवि व संगीतज्ञ हुए।

सातवां स्वर निषाद है जिसकी उत्पत्ति सहस्त्रार चक्र से हुई है। सहस्त्रार चक्र का स्थान सिर के उपरी भाग जहां पर चोटी रखी जाती है। उस स्थान को कपाल कहा जाता है। निषाद की उपासना से व्यक्ति निरलिप्तता की अवस्था में पहुँचता है। तभी कहा भी गया है 'निरलिप्त समाधिना' अर्थात जो व्यक्ति सांसारिक भोगों को छोड़कर समाधि की अवस्था में पहुँच जाता है वह मोक्ष को प्राप्त करता है। इस अवस्था में पहुँचने के लिए ताल सहायक होती है, जब ताल में कम्पन पैदा होता है तो मूलाधार चक्र जागृत होता है। यही विशिष्ट कम्पन सभी चक्रों को जागृत करके मनुष्य निरलिप्त अवस्था अर्थात् मोक्ष मार्ग की ओर बढ़ जाता है। इस प्रकार यौगिक क्रियाओं से संगीत के द्वारा परमात्मा से सम्बन्ध स्थापित करना सम्भव है। संगीत के हर पहलु में योग का किसी न किसी रूप में प्रयोग होता है क्योंकि ध्यान, धारणा और समाधि संगीत विषय के मुख्य अंग है। ध्यान द्वारा स्वर का रूप देखा जाता है। धारणा द्वारा स्वर कितना प्रबल है, इसकी सिद्धि की जाती है तथा सामाधि द्वारा विभिन्न स्वर-लहरियों में खोकर संगीतकार एक सुन्दर संगीत की रचना करता है।

संगीत भी योग के समान व्यक्ति में आस्तिकता का प्रतिपादन करता है। सुर और चैतन्य सदृश योगियों के लिए ईष्ट इसलिए सुन्दर नहीं कि कृष्ण सुन्दर है, बल्कि वे इसलिए सुन्दर है क्योंकि वह सुर और चैतन्य की सर्वोच्च आस्था के प्रतीक हैं। आस्था के अभाव में लगाव नहीं प्रीत नही, प्रीत के अभाव में भाव-गहनता नहीं हुआ करती, भाव के बिना सुन्दरता भी नहीं होती। अतः आनन्द मात्र कपोल कल्पना ही होगा।

\section{उपसंहार}

इस प्रकार कह सकते हैं कि मन की चंचलता दूर कर उसे स्थिर बनाने वाली श्रेष्ठतम विद्या योग है। तो श्रेष्ठतम कला संगीत। इस प्रकार संगीत और योग का आपस में गहरा सम्बन्ध है। योग के समान संगीत का प्राथमिक विकास भी, आध्यात्मिक विकास के लिए हुआ है। संगीत को ईश्वर का दर्जा प्राप्त है इसलिए इस विधा में शुद्धता और शास्त्रीयता का विशेष महत्व है। सात शुद्ध और पांच कोमल स्वरों के माध्यम से मन को साध लेना ही संगीत है। एक तरफ जहां 'योग' से शरीर, मन, मस्तिष्क को साधा जाता है वहीं संगीत हमारी आत्मा को शुद्ध करता है। संगीत में स्वरों की शुद्धता पर और उसकी पवित्रता पर जोर दिया जाता है, परन्तु योगशास्त्र में आसन व मुद्राओं पर जोर दिया है। दोनों में ही स्वर व मुद्रा की श्रेष्ठता से आनन्द तथा स्वास्थ्य पाया जा सकता है। 
संगीत और योग दोनों विधाएं अलग होने के बावजूद भी ये एक-दूसरे के पूरक हैं। दोनों के मूल तत्त्व और परिणाम सार्वकालिक है। दोनों का उद्देश्य आत्मा का परमात्मा से मिलन है।

संदर्भ

सुभद्रा चौधरी शारंदेव, (2009) संगीत रत्नाकरए, राधा पब्लिकेशन दिल्ली।

शर्मा पंकजमाल (10996) सामगान उद्धभव व्यवहार एवं सिद्धान्त, साहित्य प्रकाशन होशियारपुर। गौतम चमन लाल (1987), नादयोग, संस्कृति संस्थान बरेली।

स्वात्मा राम (1972) हटयोग प्रदीपिका, ओडियार लायेबरेरी और अनुसंधान केन्द्र। 